\title{
Dynamic Pricing Decision of Cloud Service Oriented to Elastic Scaling
}

\author{
Meng Feng a, Yan Cheng, Aiqing Kou \\ East China University of Science and Technology Shanghai, China \\ azsfm1997@126.com
}

\begin{abstract}
Can elastic scaling service and dynamic pricing bring more profits to cloud service providers? If so, under what circumstances can the benefits be maximized? In this article, a decision support model based on Markov process is proposed for pricing of cloud service oriented to elastic scaling. This service is only provided to Long-term contract customers with different time sensitivities, which will be used to divide the market into four categories: no time sensitivity, low time sensitivity, high time sensitivity, and scattered time sensitivity. The purpose of this article is to show whether elastic scaling service is necessary for cloud service providers. Through the experimental simulation, it can be found that the elastic scaling service can bring more profits to the supplier when the customer's time sensitivity is high or scattered. Dynamic pricing strategy is particularly important when facing the scattered time sensitivity of customers.
\end{abstract}

Keywords: Component; Formatting; Style; Styling; Insert.

\section{Introduction}

Currently, the demand for resource capacity of some Long-term contract customers in the cloud service market fluctuates greatly over time. For example, e-commerce sites need more virtual machines to process orders when they are carrying out large promotions, but the demand is relatively small in normal time. Therefore, the cloud service provider provides the " elastic scaling " service, which is to manually or automatically adjust the number of virtual machines occupied by customers based on their requirements. The elastic scaling service allows customers to reduce the loss of subscribing insufficient resource capacity and reduce the unnecessary cost of subscribing excessive resource capacity to allow enough usage during peak time.

The SLA agreement of in Long-term contracts is used to restrict the quality of cloud service [1]. When the quality is low, the cloud service provider must pay compensation to the customers. For cloud service providers with limited resources, elastic scaling service leads to excessive resource demand in the flexible part and insufficient resources in the shared resource pool, which will lead to higher compensation costs. At the same time, it could help cloud service providers obtain additional profits by selling idle resources. In addition, the customer's resource demand for the flexible part will be affected by its pricing. Therefore, it is necessary for small cloud service providers with limited resources to discuss whether elastic scaling service is necessary to offer and how to price the flexible part of cloud service from the perspective of revenue. As the dynamic change of customer demand leads to the dynamic change of occupied resource capacity, in order to further improve the revenue, this article adopts the dynamic pricing method for the flexible part of cloud service.

The research on elastic scaling of cloud service is still in its infancy, and there are few relevant literatures at home and abroad. Among them, the technical literature includes the prediction-based elastic scaling strategy of cloud computing proposed by Tsai WT [2] and Jianzhong Xu [3]. In addition, Mehiar and Dabbagh proposed a framework of online resource management based on the elasticity of cloud service to improve the profit of cloud service providers, but the cloud-service resources in this literature were regarded as unlimited [4]. However, whether it is necessary for cloud service providers with limited resources to provide elastic scaling service has not been discussed in relevant literature. 


\section{Customer Demand Modeling}

The sales cycle $T$ is divided into $N$ decision periods, and $t$ represents the serial number of the decision period. In the whole sales cycle $T$, the increase or withdrawal of contract customers is not considered, and the total resource capacity owned by cloud service providers during this period remains as $\zeta$. It is stipulated that within the time period $t$, each client's task will arrive at most one. Assume that if a customer's current task is not completed, its next task will not arrive. In reality, tasks are automatically queued, so it is reasonable not to consider such situations.

Based on whether they are sensitive to task completion time, customers are divided into two categories. One is time-insensitive customers, who are only interested in whether the task can be completed within the specified time and will not purchase elastic scaling service. The other one is time-sensitive customers who are willing to pay extra for completing tasks ahead of time. They are the audience of elastic scaling service, which will be further subdivided below.

\subsection{Customer Resource Demand Model}

Assume that time-sensitive customers understand their own demand fluctuations. During the time period $t$, if the demand increases, the customer will request the elastic scaling service. Cloud service providers do not understand the needs of individual customers, but they can understand the overall demand distribution through market research. According to the literature [5], the customer's demand in the time period $t$ is related to the previous time period. In the time period $t$, the demand $D_{i, t}$ of customer $i$ is given by the closed form expression

$$
D_{i, t}=d_{i}+d D_{i, t-1}+X_{i, t}
$$

where $d_{i}$ represents the starting level of customer $i$ 's demand, $d$ is the inter-week correlation coefficient, and $d \in[0,1] . X_{i, t}$ is a volatility item, which obeys the normal distribution with mean value of 0 and variance of $\sigma_{i}^{2}$. In order to reduce the complexity, $d$ is set to 0 , and the parameters $d_{i}$ and $\sigma_{i}^{2}$ are not related to the individual customer, but related to the category. This article assumes that customers all belong to the same category in terms of demand. The cloud service provider can obtain the estimated values $d_{0}$ and $\sigma_{0}^{2}$ of the parameters $d_{i}$ and $\sigma_{i}^{2}$ through historical data. The customer's volatility item $X$ follows normal distribution, that is, $X \sim N\left(0, \sigma_{0}^{2}\right)$, and the customer demand is expressed as $D=d_{0}+X$.

\subsection{Market Structure based on Reserve Prices}

Through market research, the fluctuation range of the customer's reservation price for the elastic part of the cloud service $\left[v_{\text {min }}, v_{\text {max }}\right]$ can be obtained, and it can be divided into $\mathrm{K}$ discrete ranges. Let $v_{0}=v_{\min }, v_{K}=v_{\max }$, and $v_{0}<v_{1}<v_{2}<\cdots<v_{K}$, use the minimum value $v_{k-1}$ in the interval to represent the $k$-th reserve price interval $\left[v_{k-1}, v_{k}\right]$. Let $h_{k}$ denote the probability that the customer's reservation price for the elastic scaling service belongs to the $k$-th interval, then the vector $H=\left(h_{1}, h_{2}, \ldots, h_{k}\right)$ is the customer's reservation price status for the elastic part of the cloud resources. The reservation price is different due to the different time sensitivity of customers. Therefore, customers can be divided into G categories, let $b_{g}$ represent the proportion of $g$-th customers in the entire market, and $S_{M}=\left\{b_{g}\right\}$ represents the market structure. Assuming that the reservation price status $H$ of similar customers is the same, $H_{g}$ can represent the reservation price status of the $g$-th customer.

\subsection{Customer Behavior Model}

The customer first decides whether to buy according to demand, and then according to the consumer surplus theory, if the customer's consumer surplus is not less than 0 , the purchase will be 
made. In the time period $t$, when the price decision of the cloud service provider is $a_{t}$, the consumer surplus obtained by the $g$-th customer is given by the closed form expression

$$
\Delta_{g}\left(a_{t}\right)=\sum_{k=1}^{K} b_{g} \times h_{k} \times\left(v_{k}-a_{t}\right)
$$

If the consumer surplus is less than 0 (ie, $\Delta_{g}\left(a_{t}\right)<0$ ), the customer will not purchase the service; otherwise, the customer will purchase.

\section{The Pricing Model of Cloud Service based on Markov Chain}

\subsection{Long-term Contract Model}

Let $i$ be the identification of the customer. This article assumes that a customer only signs one Long-term contract, and only the customer who signed the Long-term contract can purchase elastic scaling service. Based on the literature [6], the definition of the Long-term contract is as follows:

Define the Long-term contract of the $i$-th customer as $T\left(w_{i}, p_{i}, \pi\right)$, where $T\left(w_{i}\right)$ is the capacity agreed in the Long-term contract that must be provided to the customer when the customer needs it, $T\left(\mathrm{p}_{\mathrm{i}}\right)$ is the price of renting unit capacity in unit time as stipulated in the contract, and $T(\pi)$ represents the amount of compensation per unit time when the cloud service provider cannot provide the agreed resource capacity in time.

\subsection{Dynamic Pricing Decision Model of Cloud Service Oriented to Elastic Scaling}

The problem in this article is suitable for the Markov process, so it can be established by the Markov chain defined by the four-tuple $\{\mathrm{S}, \mathrm{A}, \mathrm{P}, f\}: \pi=\left\{\left(s_{t}\right) \rightarrow a_{t} \mid S_{t} \in S, a_{t} \in A, t=1,2, \ldots, N\right\}$ to determine the price decision, so as to achieve the goal of maximizing the total profit of the cloud service provider during the sales period.

Let $j$ denote the task identifier submitted by the $i$-th customer, then $(i, j)$ can uniquely identify the task received by the cloud service provider. The cloud server will use the set $\Omega_{t}^{I N G}=\left\{\left((i, j), w_{(i, j)}\right)\right\}$ to record the running task $(i, j)$ and the required maximum resource capacity $w_{(i, j)} \cdot w_{(i, j)}=D_{t}^{i}$, where $D_{t}^{i}$ represents the demand of client $i$ at time $t$, which can be obtained randomly from the distribution function given in section 2.1.

The following describes the Markov model in detail through the four-tuple:

1) State set $S$ : State set $S_{t}=\left(V_{t}^{r}\right), V_{t}^{r}$ is the idle resource capacity of the cloud service provider at time $t$. In this article, it specifically refers to the remaining amount of CPU. When considering other requirements, it is only to increase the dimension, and it will not affect the results of this article too much.

2) Decision set A: at time $t$, cloud service provider needs to make a price decision $a_{t}\left(a_{t} \in A\right)$ in time based on the current state $s_{t}$. This article stipulates that the price $a_{t}$ can only take one value in the discrete value set $\Phi$, where $\Phi=\{0.0,0.1,0.2, \ldots, 0.8,0.9\}$.

3) State transition process P:According to literature [7], this article sets the arrival rate and departure rate of the task into probabilities $\lambda_{a}$ and $\lambda_{l}$.

During the time period $t$, the status of the idle resource capacity may undergo the following transitions:

(1) When a new task $(i, j)$ arrives and the cloud service provider adopts decision $a_{t}$, the task set $\Omega_{t}^{I N G}$ changes to $\Omega_{t+1}^{I N G}=\Omega_{t}^{I N G}+\left\{\left((i, j), D_{t}^{i}\right)\right\}$. If $\sum_{g=1}^{G} b_{g} \times \aleph\left(\Delta_{g}\left(a_{t}\right)\right) \times\left(D_{t}^{i}-\tau\left(w_{i}\right)\right)>0$, the state $\mathrm{V}_{t}^{r}$ changes to $\mathrm{V}_{t+1}^{r}=\mathrm{V}_{t}^{r}-D_{t}^{i}$, otherwise change to $\mathrm{V}_{t+1}^{r}=\mathrm{V}_{t}^{r}-7\left(\mathrm{w}_{\mathrm{i}}\right)$.

(2) When a task $(i, j)$ leaves, the task set $\Omega_{t}^{I N G}$ changes to $\Omega_{t+1}^{I N G}=\Omega_{t}^{I N G}-\left\{\left((i, j), w_{(i, j)}\right)\right\}$. If $\sum_{g=1}^{G} b_{g} \times \aleph\left(\Delta_{g}\left(a_{t}\right)\right) \times\left(w_{(i, j)}-T\left(w_{i}\right)\right)>0$, the state $\mathrm{V}_{t}^{r}$ changes to $\mathrm{V}_{t+1}^{r}=\mathrm{V}_{t}^{r}+w_{(i, j)}$, otherwise change to $\mathrm{V}_{t+1}^{r}=\mathrm{V}_{t}^{r}+T\left(\mathrm{w}_{\mathrm{i}}\right)$. 
(3) If the price decision $a_{t}$ for the elastic part of the cloud service makes the customer's consumer surplus less than 0 (ie $\Delta_{i}\left(a_{t}\right)<0$ ), the customer will continue to use only the resource capacity in the contract. Therefore, the state $\mathrm{V}_{t}^{r}$ changes to $\mathrm{V}_{t+1}^{r}=\mathrm{V}_{t}^{r}-w_{(i, j)}+\mathrm{T}\left(\mathrm{w}_{\mathrm{i}}\right)$.

4) The income fof a state transition:The cloud service provider can obtain revenue $r_{(i, j)}^{t}$ through task $(i, j)$ running in the time period $t$, which is calculated as follows

$$
r_{t}^{(i, j)}=T\left(p_{i}\right) \times T\left(w_{i}\right)+a_{t} \times \sum_{g=1}^{G} b_{g} \times \aleph\left(\Delta_{g}\left(a_{t}\right)\right) \times\left(w_{(i, j)}-T\left(w_{i}\right)\right)
$$

where $T\left(p_{i}\right) \times T\left(w_{i}\right)$ is Long-term contract income, $a_{t} \times \sum_{g=1}^{G} b_{g} \times \aleph\left(\Delta_{g}\left(a_{t}\right)\right) \times\left(w_{(i, j)}-\right.$ $\left.T\left(w_{i}\right)\right)$ is the income obtained through elastic cloud service resources. $w_{(i, j)}$ represents the maximum resource capacity required by task $(i, j), \aleph\left(\Delta_{g}\left(a_{t}\right)\right)=\left\{\begin{array}{l}0, \Delta_{g}\left(a_{t}\right)<0 \\ 1, \Delta_{g}\left(a_{t}\right) \geq 0\end{array}\right.$ is used to determine whether the customer purchases flexible services when the decision is $a_{t}$.

The loss due to insufficient resource capacity during the time period $t$ is estimated as follows:

$$
\beta_{t}=\left\{\begin{array}{c}
\pi \times\left(\Lambda\left(-\mathrm{V}_{t}^{r} \div \mathrm{T}\left(\mathrm{w}_{\mathrm{i}}\right)\right)\right), \mathrm{V}_{t}^{r}<0 \\
0, \quad \mathrm{~V}_{t}^{r}>0
\end{array}\right.
$$

where $\Lambda(\cdot)$ represents the rounding up function.

Therefore, the benefit of a state transition is

$$
\mathrm{R}_{t}=\sum_{\left((i, j), w_{(i, j)}\right) \in \Omega_{t}^{I N G}} r_{(i, j)}^{t}-\beta_{t}
$$

Then, the objective function can be expressed as

$$
\max _{\pi \in \Pi} E\left\{\sum_{t=1}^{T^{\text {horiz }}-1} \mathrm{R}_{t}\left(\mathrm{~S}_{t}, x_{t}\right)\right\}
$$

Let $V_{t}\left(s_{t}\right)$ denote the cumulative revenue of the system from the beginning of time $t$ to the end of the sales cycle in the state $s_{t}$. The dynamic pricing decision problem of cloud service oriented to elastic scaling is to find the optimal decision chain according to the state $s_{t}$ of each unit time period $t: \Gamma=\left\{s_{t} \rightarrow a_{t}\right\}$, so that the return function described by the following Bellman recursive equation takes the maximum value.

$$
\mathrm{V}_{t}\left(\mathrm{~s}_{t}\right)=\max _{x_{t}}\left(\mathrm{R}_{t}\left(\mathrm{~s}_{t}, a_{t}\right)+E\left\{\mathrm{~V}_{t+1}\left(\mathrm{~s}_{t+1}\right) \mid \mathrm{s}_{t}\right\}\right)
$$

\section{Experiment and Result Analysis}

\subsection{Q-learning}

In this paper, Q-learning technology is used to simulate this kind of Markov decision problem. The training result of Q-learning is a Q-value table based on state and decision. When making a decision, it is enough to find the decision $a$ that maximizes the $\mathrm{Q}$ value in the $\mathrm{Q}$ value table according to the current state, so the algorithm can meet the real-time requirements of the decision.

In the simulation learning process, the agent observes the state $s_{t}$ at each time step $t$ and executes a certain decision $a_{t}$. After the environment accepts the decision, a state transition $s_{t} \rightarrow s_{t+1}$ occurs, 
and it receives an instant reward $f\left(s_{t}, a_{t}\right)$, and then according to $\mathrm{Q}\left(s_{t}, a_{t}\right)=(1-\beta) \times \mathrm{Q}\left(s_{t}, a_{t}\right)+$ $\beta \times\left(\mathrm{f}\left(s_{t}, a_{t}\right)+\gamma \times \mathrm{V}\left(s_{t+1}\right)\right)$, where $\mathrm{V}\left(s_{t+1}\right)=\max _{a \in A}\left(Q\left(s_{t+1}, a\right)\right), \beta$ is the learning rate parameter, and $\gamma$ is the time discount rate of income. After a large number of iterations, the Q-values of various $(s, a)$ combinations will tend to converge, and then the Q-learning training is completed.

\subsection{Case Analysis}

In the experiment, the learning rate $\beta$ is set to 0.6 and the discount factor $\gamma=0.9$. Assume that the sales cycle contains 50 unit time periods. Customers are divided into the following 4 categories as shown in Table 1. In order to show the choice of cloud service providers in different types of customer markets, four extreme customer markets were selected, as shown in Table 2. For the simplicity of the experiment, in this experiment, the total capacity available of cloud service provider is equal to the sum of capacity set in the the Long-term contract of all customers, and Long-term contract $T\left(w_{i}, p_{i}, \pi\right)$ is set by the parameters $T(1,0.5,0.1)$.

Table 1. Preference parameters of customers' purchasing behavior

\begin{tabular}{|c|c|c|}
\hline Customers'type & Reserve price $\left(v_{i}\right)$ & Demand fluctuation status $\left(d_{i}, e_{i}^{2}\right)$ \\
\hline$A$ & 0 & \multirow{2}{*}{$(0,1)$} \\
\hline$B$ & 0.3 & \\
\hline$D$ & 0.6 & \\
\hline
\end{tabular}

Table 2. Customer Market Distribution

\begin{tabular}{|c|c|c|}
\hline The number of markets' type & Market type & Customers' type distribution \\
\hline 1 & Evenly distributed customer types & $\{0.25,0.25,0.25,0.25\}$ \\
\hline 2 & Low customer time sensitivity & $\{0.5,0.5,0,0\}$ \\
\hline 3 & Customer time sensitive type & $\{0,0,0.5,0.5\}$ \\
\hline 4 & Only time-insensitive client type & $\{1,0,0,0\}$ \\
\hline
\end{tabular}

Figure 1 shows the total profit that cloud service providers can obtain in different kinds of markets during the iterative process. It can be found that implementing elastic scaling services in a market with high customer time sensitivity can achieve the highest profit. Because customers are relatively time sensitive, and then the pricing of elastic services can be increased. In addition, as the proportion of highly time-sensitive customers decreases, the additional benefits of elastic scaling gradually decline. In a time-insensitive customer market, elastic scaling is unnecessary for cloud service providers, and they may even pay more for development. When the number of iterations is small, the profit for the market with low customer time sensitivity is also relatively low, indicating that under fixed pricing, it is not recommended to use elastic scaling in the market with low customer time sensitivity. For markets where customers are relatively time-sensitive or dispersed, it is recommended to provide flexible scaling regardless of whether dynamic pricing is adopted.

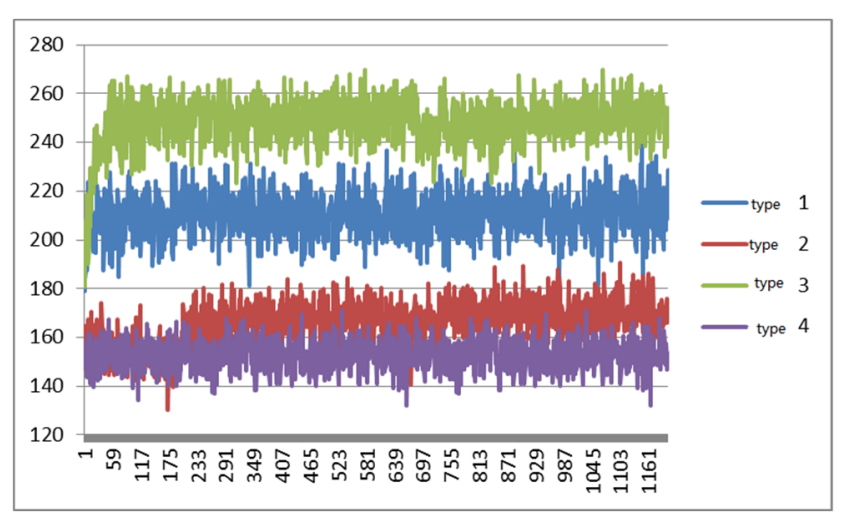

Figure 1. Profits can be obtained under 4 market types under different iteration times 
Figure 2 shows the dynamic pricing given by cloud service providers for the elastic part of the service under different idle resource capacities. What is missing is that the elastic scaling service is closed in this case. Only in the time-insensitive customer market, elastic scaling will be turned off regardless of the amount of idle resource capacity, which is consistent with common perceptions. In a market with low customers time sensitivity, it is recommended to turn off elastic scaling service when the idle capacity is only about half or very small.

The former is because each customer will not submit any tasks when there is task in progress. When the idle capacity is only about half, the uncertainty of how many tasks will arrive in the next time period is large and half of the capacity is already occupied, if you set elastic scaling service, the risk of default compensation is relatively high. The latter is because there is very little free capacity, the probability of accepting default payments is extremely high, and the customer's time sensitivity is low, and the extra profit obtained is less than default payments. In a market with high customer time sensitivity, since most customers are willing to pay extra fees for flexible services even higher than the original contract fees, elastic scaling service is provided regardless of the amount of idle capacity.

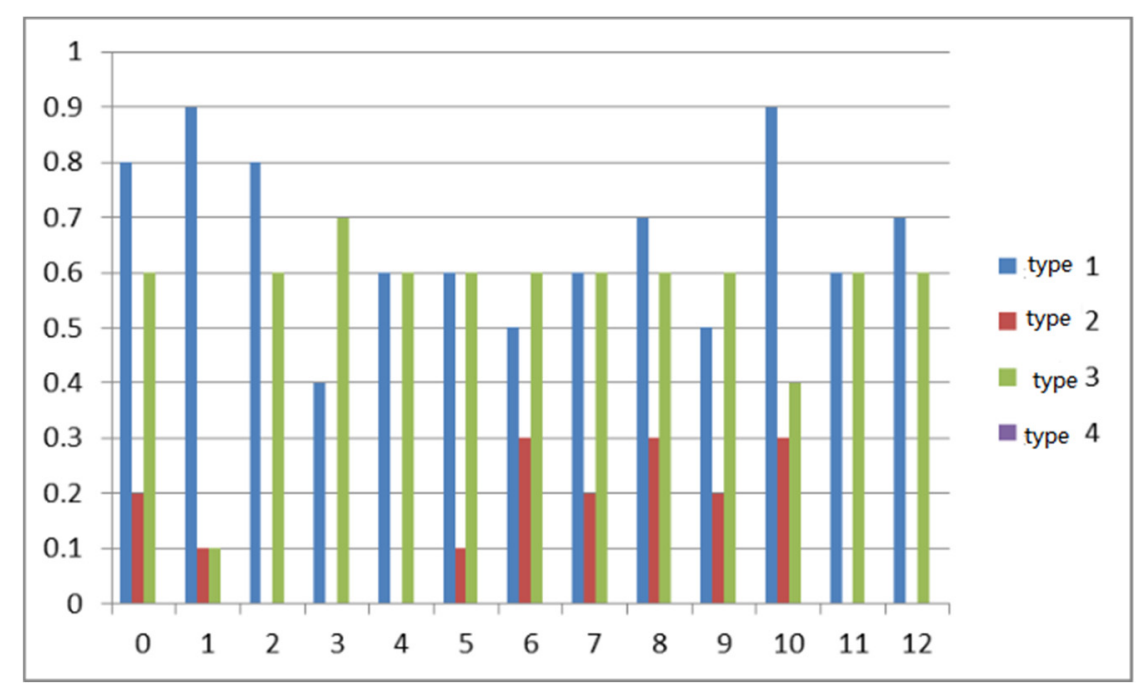

Figure 2. Actual pricing of different idle resource capacities in different markets

\section{Conclusion}

In order to further improve the revenue of cloud service providers, this article establishes a pricing model for elastic scaling service based on the Markov process, and then simulates, solves, and analyzes the model through the $\mathrm{Q}$ learning algorithm. The pricing decisions and total revenue of elastic scaling service in four markets based on different customer time sensitivity are given. Experimental results show:

(1) For markets with even distribution of customer types or high customer time sensitivity, elastic scaling service is recommended.

(2) Markets with high customer time sensitivity can adopt fixed pricing, but the benefits of dynamic pricing are higher; While in the market with evenly distributed customer types, dynamic pricing must be adopted.

This article is a study on whether to provide elastic scaling service for Long-term contract customers in four market situations. However, this article doesn't predict the fluctuations of the customer's resource demand over time. At the same time, it didn't consider other losses when the SLA agreement is violated, such as the customers may refuse to sign the contract again. These can be further studied in the future. 


\section{References}

[1] Legal issues in clouds: towards a risk inventory[J]. Philosophical Transactions: Mathematical, Physical and Engineering Sciences,2013,371(1983).

[2] Tsai W T, Huang Y, Shao Q.2011.Testing the scalability of SaaS applications.

[3] Xu Jianzhong, Wang Jun, Zhou Xunzhao, Xu Lei. 2018. A forecast-based elastic scaling strategy for cloud computing. Computer and Digital Engineering. 46 (06): 1160-1162+1231.

[4] Mehiar Dabbagh, Bechir Hamdaoui, Mohsen Guizani. 2015. Exploiting Task Elasticity and Price Heterogeneity for Maximizing Cloud Computing Profits. IEEE Transactions on Emerging Topics in Computing.

[5] X Qin, Jing L I, Yuan Y, et al. Customer Environment Demand Forecasting Method Based on Gray Markov Model [J]. Machine Design \& Research, 2018.

[6] Yuan S, Das S, Ramesh R, et al. Service Agreement Trifecta: Backup Resources, Price and Penalty in the Availability-Aware Cloud [J]. Information Systems Research, 2018, 29(4):947-964.

[7] Hong Xu 0001, Baochun Li. Dynamic Cloud Pricing for Revenue Maximization. [J]. IEEE Trans. Cloud Computing, 2013, 1(2). 